\title{
Virtual Biobanking for Retroperitoneal Sarcoma: A Transatlantic Australasian Retroperitoneal Sarcoma Working Group (TARPSWG) Initiative
}

\author{
Eisar Al-Sukhni, MD, MSc ${ }^{1}$, Winan J. van Houdt, MD, $\mathbf{P h D}^{2}$, Sally M. Burtenshaw, MSc ${ }^{1}$, \\ Yael Babichev, $\mathrm{PhD}^{1}$, Chandrajit P. Raut, MD, MSc ${ }^{3}$, Marco Fiore, $\mathrm{MD}^{4}$, Elizabeth G. Demicco, MD, $\mathrm{PhD}^{5}$, \\ Alessandro Gronchi, $\mathrm{MD}^{4}$, and Rebecca A. Gladdy, $\mathrm{MD}, \mathrm{PhD}^{1}$ \\ ${ }^{1}$ Surgical Oncology, Princess Margaret Cancer Centre, Mount Sinai Hospital, Department of Surgery, University of \\ Toronto, Toronto, ON, Canada; ${ }^{2}$ Surgical Oncology, Netherlands Cancer Institute, Amsterdam, The Netherlands; ${ }^{3}$ Surgery, \\ Brigham and Women's Hospital, Dana-Farber Cancer Institute, Harvard Medical School, Boston, MA; ${ }^{4}$ Surgery, \\ Fondazione IRCCS Istituto Nazionale dei Tumori, Milan, Italy; ${ }^{5}$ Department of Pathology and Laboratory Medicine, \\ Mount Sinai Hospital, Department of Laboratory Medicine and Pathobiology, University of Toronto, Toronto, ON, Canada
}

Sarcomas are rare tumors comprising more than 70 distinct histologic types. The broad spectrum of behavior of these tumors, in addition to the lack of effective standard therapeutic agents for most, are factors underlying the need for translational research in this field. Biobanking-the collection of biomaterials as well as associated clinical, pathologic, and molecular data-facilitates translational research by providing the material not only to investigate the molecular contributors of tumor pathogenesis but also to develop new treatments and investigate their effectiveness on an individual level (i.e., personalized medicine). ${ }^{1}$ Pooling resources from multiple biobanks can further this goal by providing a larger foundation of specimens on which to build translational research. ${ }^{2}$ For diseases, such as sarcoma, that consist of numerous rare distinct types, collaboration is critical to achieve sufficient numbers to define molecular mechanisms and to develop prognostic biomarkers.

Electronic supplementary material The online version of this article (https://doi.org/10.1245/s10434-020-08775-1) contains supplementary material, which is available to authorized users.

(C) Society of Surgical Oncology 2020

First Received: 18 April 2020;

Published Online: 26 June 2020

R. A. Gladdy, MD, PhD

e-mail: rebecca.gladdy@ sinaihealth.ca
The Transatlantic Australasian Retroperitoneal Sarcoma Working Group (TARPSWG) was established in 2013 with the goal to evaluate the current evidence on retroperitoneal sarcoma and to develop consensus guidelines. ${ }^{3}$ Current membership encompasses 119 institutions across Europe, North America, Central America, Asia, and Australia. Members are clinicians and scientists with interest in the management of retroperitoneal sarcoma, although the majority also treat sarcomas that arise in other sites. Many are affiliated with high-volume sarcoma centers, which are regional and national centers of excellence.

We sought to describe the current state of sarcoma biobanking amongst high-volume sarcoma centers worldwide and to gauge interest in collaboration. Members of TARPSWG were surveyed to define current biobanking efforts and long-term goals. Our goals were 1) to assess the current state and standards of practice for biobanking across TARPSWG institutions, and 2) to determine whether members thought centralized biobanking would be sensible and feasible to facilitate translational research.

\section{MATERIALS AND METHODS}

An English-language survey was made available online using a web-based survey service (SurveyMonkey Inc., San Mateo, CA). Twenty-one questions were posed that focused on current clinical data collection, biobanking procedures, and level of interest to collaborate. 
Current members of TARPSWG as identified through the 2018 membership list were invited to participate. Initial surveys were sent in February 2018. Reminders were sent twice by email and responses were collected until May 2018. Data analysis was undertaken following survey closure. Data were summarized using descriptive statistics.

\section{RESULTS}

Email invitations were sent to 134 TARPSWG members from 58 institutions, which was the TARPSWG member institutions at the time of the survey. Forty-nine members from 42 centers completed the survey, which represented $71 \%$ of centers with TARPSWG membership. Respondents were from 14 countries across 4 continents with demographic and practice details summarized in Table 1. The majority of respondents were surgical oncologists (76\%), and more than $58 \%$ had clinical practices composed of at least $50 \%$ sarcoma. Three-quarters of respondents reported managing sarcomas at several body sites (abdomen/pelvis, retroperitoneum, trunk, and extremity).

Most $(80 \%)$ indicated that their institutions had an ethics board-approved prospective sarcoma clinical database. Databases were established between 1995 and 2018 and accrued a range of 10-500 patients per annum. The majority of centers were undertaking biobanking (88\%)-a practice established as early as 1998 in one center. Almost all centers $(97 \%)$ stored their surgical specimens as formalin-fixed, paraffin-embedded (FFPE) and/or fresh frozen samples, whereas only $18 \%$ also stored germline tissue (Table 2). Biobanks were housed within the institution in

TABLE 1 Demographic and practice details of survey respondents

\begin{tabular}{lrr}
\hline & Number of respondents & $\%$ \\
\hline Primary practice & 37 & 76 \\
Surgical oncology & 4 & 8 \\
Medical oncology & 4 & 8 \\
Radiation oncology & 3 & 6 \\
Pathology & 1 & 2 \\
Basic research & & \\
Proportion of practice (sarcoma) & 7 & 15 \\
$<$ 25\% & 13 & 27 \\
25-50\% & 14 & 29 \\
51-75\% & 14 & 29 \\
$>$ 75\% & & \\
Sites managed clinically & 36 & 77 \\
Abdo/pelvis/trunk/extremity & 6 & 8 \\
Abdo/pelvis/trunk (including breast) & 4 & 2 \\
Abdo/pelvis only & 1 & \\
Other & &
\end{tabular}

nearly all cases $(97 \%)$ and were managed centrally by pathology in $55 \%$, directly by clinical faculty in $40 \%$, and centrally by the government in $5 \%$. Funding sources for biobanking varied worldwide: $74 \%$ reported having institutional funding, whereas three relied solely on philanthropy and four on grants. Although $95 \%$ of respondents reported biobanking pretreated sarcoma specimens, standardized operating procedures to ensure tissue quality of the samples were not uniform. Most centers (78\%) performed pathologic review of specimens, but only $34 \%$ performed quality checks of RNA or DNA quality. Other quality assurance measures reported were the use of CAP certified biorepository ( 1 center) and sample QC at the time of use for research ( 1 center). Lack of funding, limited infrastructure, and legal/ethical considerations were perceived barriers for centers that did not routinely biobank (Table 2).

The majority of respondents were willing to share biobanked specimens with TARPSWG members (89\%), depending on the protocol proposed. Overall, a virtual biobank with tissue storage at local centers was preferred over a centralized TARPSWG biobank.

\section{DISCUSSION}

This survey establishes a snapshot of current sarcoma biobanking practices worldwide and canvassed the interest of sarcoma experts regarding collaborative international biobanking. We achieved an excellent response rate of $71 \%$, and thus, these data are representative of the state of biobanking across high-volume sarcoma centers globally.

While most institutions sampled are making some effort toward biobanking, there is variability in operating procedures related to ensuring specimen quality. This may limit the utility of sharing specimen data across centers. The challenge of inadequate quality assessment of biobanked specimens was highlighted in a systematic review of measures that researchers used to evaluate tissue specimen quality. ${ }^{4}$ Parameters reported as surrogates for biospecimen integrity, such as storage time or time to cryopreservation, were not effective indicators of quality control and assurance. Although RNA integrity is generally accepted as a measure of specimen qualification, the authors reported that fewer than half of studies included assessed the reliability of RNA for downstream applications.

Several organizations have published guidelines for standard operating procedures (SOPs). In Canada, the Canadian Tissue Repository Network (CTRNet) provides biobanking guidelines SOP's generation, which include specimen quality assessment, facility management/operation, and clinical annotation and document maintenance. ${ }^{5}$ Specimen annotation is critical in the creation of a 
TABLE 2 Biobanking practices and opinions of TARPSWG institutions surveyed

\begin{tabular}{|c|c|c|}
\hline & Number of respondents & $\%$ \\
\hline Centers performing biobanking & 42 & \\
\hline Types of specimens banked (multiple answers permitted) & 38 & \\
\hline Operative specimens & 37 & 97 \\
\hline Biopsy & 18 & 47 \\
\hline Germline tissue & 7 & 18 \\
\hline Mode of storage (multiple answers permitted) & 37 & \\
\hline Fresh frozen & 35 & 95 \\
\hline FFPE & 30 & 81 \\
\hline Cell lines & 7 & 19 \\
\hline Other & 2 & 5 \\
\hline Tissue housed at institution & 38 & \\
\hline Yes & 37 & 97 \\
\hline Funding for biobank & 34 & \\
\hline Institution & 25 & 73 \\
\hline Grant & 4 & 12 \\
\hline Philanthropy & 3 & 9 \\
\hline Other & 2 & 6 \\
\hline Management of biobank & 38 & \\
\hline Centrally (i.e. pathology) & 21 & 55 \\
\hline Clinical faculty & 15 & 39 \\
\hline Centralized by government & 2 & 5 \\
\hline Standard operating procedures in place (multiple answers permitted) & 32 & \\
\hline Pathologic review of specimen & 25 & 78 \\
\hline Quality checks of extracted RNA or DNA quality & 11 & 34 \\
\hline Other & 5 & 16 \\
\hline Specimens from patients given neoadjuvant therapy excluded from biobank & 37 & \\
\hline Yes & 1 & 3 \\
\hline \multicolumn{3}{|l|}{ Willing to share biobanked specimens for TARPSWG research protocols } \\
\hline Yes & 15 & 39 \\
\hline Possibly, with IRB approval & 4 & 11 \\
\hline Answer dependent on protocol interest & 19 & 50 \\
\hline \multicolumn{3}{|l|}{ Barriers to biobanking among centers not biobanking } \\
\hline Lack of funds & 3 & 33 \\
\hline Limited infrastructure & 1 & 11 \\
\hline Legal and ethical regulations & 2 & 22 \\
\hline Other & 3 & 33 \\
\hline \multicolumn{3}{|l|}{ Interest in centralized tumor banking for TARPSWG } \\
\hline Yes, in addition to own biobank & 27 & 61 \\
\hline Yes, instead of own biobank & 1 & 2 \\
\hline No & 10 & 23 \\
\hline Uncertain & 6 & 14 \\
\hline
\end{tabular}

"virtual" biobank, as data acquired at one site may be shared with a larger group without direct access to the original specimen for data interrogation. Thus, we generated and provide SOPs (Online Appendix) for the collection of tissue and blood as well as relevant collection forms as part of this study.
A second issue is that only a minority (18\%) of centers are currently biobanking germline material. This was not surprising given the additional labor and cost of collecting and banking normal tissue. The utility of germline material collected in conjunction with tumor specimens is critical as a matched internal control, which aids in distinguishing somatic versus germline mutations. In sarcoma, while 
somatic mutations are identified in a variety of histologic types, only a small fraction of diagnosed cases are attributed to a known hereditary predisposition. ${ }^{6}$ However, as Ballinger and colleagues demonstrated in the International Sarcoma Kindred Study, amongst a cohort of 1162 patients with sarcoma recruited from specialist clinics regardless of family history, about half bore an excess of pathogenic germline variants in known and novel cancer genes, although only $17 \%$ of the cohort fit into recognizable family syndromes. ${ }^{7}$ This prevalence of germline mutations suggests the need to collect germline tissue to define the genetic mechanisms contributing to sarcoma development. This issue is an important consideration in the SOPs for biobanking supported by the TARPSWG group.

Our study revealed that there is widespread interest among TARPSWG membership in centralized virtual tumor banking as well as a general willingness to share specimens for research protocols that are supported by the group. However, some barriers to specimen biobanking identified were lack of funding and limited infrastructure. The financial challenge of establishing and maintaining a biobank is a practical problem that some biobanks have addressed by charging investigators for access to specimens and related data. Despite this, the majority of biobanks continue to rely on public and/or private contributions for their maintenance. ${ }^{8}$ It is conceivable that integrating user fees into a virtual biobank will aid in increasing funding to each biobank, while allowing a larger pool of researchers access to valuable specimen resources.

In addition to quality control and financial considerations, a major potential challenge facing multinational biobanking collaboration is the variability in ethical and legal regulations between participating centers. Within the European Union, for example, the General Data Protection Regulation (GDPR) was established in May 2018 and provided directives regarding transparency and accountability to data subjects as well as strengthening requirements for informed consent of participating subjects. ${ }^{9}$ Under this regulation, international data sharing via a virtual biobank is restricted by the requirement that collaborating institutions must all meet the standards for data processing and protection mandated by the European Union. For countries that do not meet these standards, additional "appropriate safeguards" are required, which must be "binding and enforceable" in the country in question. ${ }^{10}$ However, these challenges can be navigated by TARPSWG collaborators as they continue to harmonize consent and data sharing to facilitate this multinational endeavor, which is needed to characterize more comprehensively these rare tumors.

\section{CONCLUSIONS}

The TARPSWG collaboration to date has focused on clinical management and outcomes in retroperitoneal sarcoma patients, which has expanded to more than 14 countries worldwide. Virtual biobanking for translational research projects is enthusiastically supported by TARPSWG members, which will ensure large availability of rare tumors. We present standard operating procedures for biobanking while ongoing efforts are needed to ensure specimen quality.

ACKNOWLEDGMENTS The authors thank the participants and ongoing collaborations of our colleagues in TARPSWG.

DATA AVAILABILITY STATEMENT The full data that support the findings of this study are available from the corresponding author upon reasonable request.

\section{REFERENCES}

1. Liu A, Pollard K. Biobanking for personalized medicine. Adv Exp Med Biol. 2015;864:55-68.

2. Patil S, Majumdar B, Awan KH, et al. Cancer oriented biobanks: a comprehensive review. Oncol Rev. 2018;12:357.

3. Transatlantic Australasian Retroperitoneal Sarcoma Working Group. (2019) About Us. Available at: https://tarpswg.org/infor mazioni/.

4. Caixeiro NJ, Lai K, Lee CS. Quality assessment and preservation of RNA from biobank tissue specimens: a systematic review. $J$ Clin Pathol. 2016;69:260-5.

5. Canadian Tissue Repository Network. Standard Operating Procedures. 2019. Available at: https://www.ctrnet.ca/resources/ope rating-procedures.

6. Farid M, Ngeow J. Sarcomas associated with genetic cancer predisposition syndromes: a review. Oncologist 2016;21: 1002-13.

7. Ballinger ML, Goode DL, Ray-Coquard I, et al. Monogenic and polygenic determinants of sarcoma risk: an international genetic study. Lancet Oncol. 2016;17:1261-71.

8. Coppola L, Cianflone A, Grimaldi AM, et al. Biobanking in health care: evolution and future directions. J Transl Med. 2019;17:172.

9. Regulation (EU) 2016/679 of the European Parliament and of the Council of 27 April 2016 on the protection of natural persons with regard to the processing of personal data and on the free movement of such data, and repealing Directive 95/46/EC (General Data Protection Regulation), 2016.

10. BBMRI-ERIC. The EU General Data Protection Regulation, Answers to Frequently Asked Questions. 2017. Available at: h ttp://www.bbmri-eric.eu/wp-content/uploads/BBMRI-ERIC_FA Qs_on_the_GDPR_V2.0.pdf.

Publisher's Note Springer Nature remains neutral with regard to jurisdictional claims in published maps and institutional affiliations. 